\title{
Glucose fluctuation Impact on Delayed Cerebral Infarction and 30 Days Mortality in Aneurysm SAH: A Retrospective Study in Neurocritical Care Unit
}

\section{Qian Wu}

Department of Neurology, First Affiliated Hospital, Kunming Medical University, Kunming, Yunnan,China.

\section{Liyuan Tao}

Research center of cilinical epidemiology,Peking university Third Hospital,Beijing, China

\section{Lianmei Zhong}

Department of Neurology, First Affiliated Hospital, Kunming Medical University, Kunming, Yunnan,China.

\section{Li-ping Liu}

Department of Neurology, Beijing Tiantan Hospital, Capital Medical University, Beijing, China

Donghua Mi ( $\sim$ midonghua131@163.com )

Department of Neurology, Beijing Tiantan Hospital, Capital Medical University, Beijing, China

\section{Research Article}

Keywords: aSAH, Glucose Fluctuation, DCl, Mortality

Posted Date: February 17th, 2021

DOI: https://doi.org/10.21203/rs.3.rs-199960/v1

License: (c) (i) This work is licensed under a Creative Commons Attribution 4.0 International License. Read Full License 


\section{Abstract}

Background Hyperglycemia was associated with delayed cerebral infraction (DCl) and poor outcomes in aneurysmal subarachnoid hemorrhage (aSAH) patients. However, the influence of glucose fluctuation on $\mathrm{DCl}$ and mortality in aSAH patients was still unclear.

Objective This study aimed to clarify the relationship of glucose fluctuation and $\mathrm{DCl}$ occurrence or 30 days mortality in aSAH patients who were admitted in Neurocritical Unit (NCU).

Methods A total of 341 patients with aSAH were retrospectively recruited and studied. Continuous 14-day fasting blood glucose was collected and divided into four groups: stable, unstable, well-controlled, bad-controlled. Risk factors that were associated with glucose fluctuation, $\mathrm{DCl}$ occurrence and 30 days mortality was analyzed.

Results Many risk factors, such as Age (59.09 \pm 11.95$)$, Diabetes history, SBP $(162.91 \pm 20.85)$, BUN $(5.50 \pm 1.80)$, HA1c (7.12 \pm 1.65$),$ BGV $(0.32 \pm 0.11)$, Hunt-Hess Score (2.59 \pm 0.97$)$, GCS Score (10.68 \pm 4.23$)$, Hydrocephalus in Forth ventricle, Third ventricle and Lateral ventricles, are associated with glucose fluctuation. Bad controlled group has the highest mortality $(15.91 \%)$, then unstable $(13.04 \%)$, well-controlled $(6.45 \%)$, and stable group $(1.42 \%)$. The unstable group has the highest incidence of $\mathrm{DCl}(39.13 \%)$, then bad-controlled $(29.55 \%)$, the stable $(17.92 \%)$ and well-controlled (17.74\%).

Conclusions Unstable and bad-controlled glucose during NCU admission was associated with $\mathrm{DCl}$ and 30 days mortality of aSAH patients.

\section{Introduction}

Subarachnoid hemorrhage (SAH), a life-threatened disease, is often caused by rupture of cerebrovascular aneurysm. Cumulative case fatality rates in the natural history of SAH over time are as follows: first day, 25-30\%; first week, $40-45 \%$; first month, $50-60 \%$; sixth month, $55-60 \%$; first year: $65 \%$ and fifth year, $65-70 \%$. Approximately $12 \%$ of patients die before reaching medical attention. Many risk factors, such as early brain injury, delayed cerebral ischemia $(\mathrm{DCl})$, increasing sympathetic activity, hyperglycemia, hypoglycemia and dysnatremia are associated with poor outcomes after $\mathrm{SAH}[1]$. As glucose is metabolic substrate of central nervous system, both hypoglycemia and hyperglycemia would have equally worse outcomes[2], such as DCl and increased risk of pneumonia, which could lead to increased length of stay of ICU-days or hospital-days, even high mortality of aSAH in-hospital[2, 3]. Thus, several studies focused on how to control glucose level in neurological diseases, including stroke, traumatic brain injury (TBI), SAH, and even severe sepsis in Neurocritical Care Unit (NCU). The European Stroke Organization guidelines recommend that hyperglycemia with blood glucose $>10 \mathrm{mmol} / \mathrm{L}(180 \mathrm{mg} / \mathrm{dL})$ should be treated [4]. However, a strict glucose control regimen seemed failed to improve time trend-adjusted neurological outcomes or reduce mortality after SAH [1]. Meanwhile, extensive changes in blood glucose concentrations, which is to say, big glucose variability was also associated with cerebral metabolic distress [5], energy metabolic crisis, and an elevated lactate/pyruvate ratio [6], which would finally result in unfavorable outcomes $[5,6]$. Thus, moderate glucose control and minimizing glucose variability are important concepts in glycemic management in SAH patients, but the optimal target range remains unknown. Meanwhile, no consensus has been reached on the suitable glycemic level after $\mathrm{SAH}$, and few studies have addressed this topic in terms of glucose, glucose variability and mortality in SAH. 
$\mathrm{DCl}$ was a serious complication that gave rise to poor recovery and clinical outcomes, such as hemiparesis, confusion or drowsiness, persistent cognitive impairment, social and emotional disorders, in survivors of aneurysmal subarachnoid hemorrhage (aSAH) [7]. DCI was mainly believed associated with early brain injury, impaired cerebral metabolism, regional or global hypoperfusion in brain, and cerebral arterial vasospasm [8]. Besides, more clinical and radiological features were also involved in $\mathrm{DCl}$ incidence. Smoking was the strongest risk factor of $\mathrm{DCl}$. Other possible factors included diabetes mellitus (DM), hyperglycemia on admission, acute hydrocephalus, early systemic inflammatory response syndrome, female, hypertension, CT Fisher grade and age [9]. DCl occurred after aSAH seemed have a complicated regulatory mechanism.

Both DM and hyperglycemia were considered as predictors and risk factors of $\mathrm{DCl}$ occurrence in aSAH. A twocenter retrospective cohort study indicated that maximum glucose level $(8.6-11.8 \mathrm{mmol} / \mathrm{L})$ within the first 24 hours after admission may be as a consequence of increased adrenergic stress and as independent factor of higher DCl suffering in critically aSAH patients[10]. Moreover, glucose, as a direct precursor of lactate, its rise will increase the circulating levels of lactate which was an independent predictor of poor outcome of aSAH[10]. Additionally, Hyperglycemia was proven to be associated with vasospasm by inhibition of vasodilatation and enhancement of vasoconstriction, with thrombin formation by increase of coagulation and decrease of fibrinolysis, with increase of proinflammatory transcription factors and proinflammatory cytokines, all of which are possibly the pathophysiological causes of DCI[2]. Furthermore, Hyperglycemia can also facilitate the conversion of ischemia to irreversible infarction[11]. Hence, glucose management was obviously important to avoid occurrence of $\mathrm{DCl}$ and ameliorate clinical outcome after aSAH. Nowadays, most evidence focused on acute glucose level and its relationship to $\mathrm{DCl}$ incidence. However, for critically ill patients, due to stress response to disease itself or surgery, glucose level might be persistently high or fluctuated strongly after aSAH for several days, or even weeks without intervention. Persistent hyperglycemia, other than a single hyperglycemia event, was proven to be independent predictors of postoperative outcomes, which is 10 -fold more likely to have a poor 2-week outcome and sevenfold more likely to have a poor outcome 7 to 13 months after aSAH[12]. Nevertheless, little evidence had shown that whether persistent hyperglycemia or glucose fluctuation might predict $\mathrm{DCl}$ occurrence and how to modulate glucose level might prevent critically ill patients with aSAH from suffering $\mathrm{DCl}$.

Here, we hypothesized that persistent hyperglycemia or abnormal glucose fluctuation might be associated with occurrence of $\mathrm{DCl}$ and 30 days mortality in aSAH patients. Our study retrospectively recruited aSAH patients in NCU, collected their 14 days continuous glucose level from their first day on admission and focused on the impact of glucose variation on $\mathrm{DCl}$ incidence and 30 days mortality.

\section{Methods}

\subsection{Subjects}

Patients were recruited from a retrospectively collected database of all patients with SAH who were admitted to Tiantan Hospital in Beijing, China from January 2015 and December 2017. All patients suffered $\mathrm{f}$

\subsection{Subjects}

Patients were recruited from a retrospectively collected database of all patients with SAH who were admitted to Tiantan Hospital in Beijing, China from January 2015 and December 2017. All patients suffered from aneurysm SAH were included. People with SAH caused by vasculitis, trauma, or rupture of an arteriovenous malformation was excluded. There were 341 subjects (148 males, $43.4 \%$; 193 females, $56.6 \%$ ) were enrolled in this study. We 
have two experts to evaluate the occurrence of $\mathrm{DCl}$, the Kappa value was 0.91 between the two experts. This retrospective study was approved by the Ethics Committee at the Tiantan Hospital in Beijing, in accordance with the Declaration of Helsinki. Our study was approved to collect data without requiring patient informed consent under the Waived Consent from the Ethics Committee at the Tiantan Hospital in Beijing (Ethics Approval Number: KY2019-060).

\subsection{Clinical information}

The following baseline characteristics were analyzed based on the data collected during the period of admission: sex, age, diabetes mellitus (DM), hypertension, glucose level, initial Glasgow Coma Scale (GCS) score, systolic blood pressure (SBP), diastolic blood pressure (DBP), temperature, treatment, surgery, mortality information and dates of death. Several indexes were collected as follows: AST, ALT, BUN, creatinine (Cr), D-dimer, BNP, EF, hemoglobin A1c (HA1c), blood glycemic variability (BGV), Hunt Hess, CT Fisher, hydrocephalus (Fourth ventricle, Third ventricle, Lateral ventricle, Quadrigeminal cistern, Ambiens cisterna, Suprasellar cistern, Sylvian fissure, Basilar cistern). The first day blood glucose level was taken immediately before any glucose management when admission. Then the other 13 days blood sample was taken around 6:00 am every morning as fasting blood glucose. These 14 days glucose value after SAH were collected and was divided into four groups - stable, unstable, well-controlled and bad controlled (Table 1). BGV was measured as glycated albumin/glycosylated hemoglobin ratio[13].

\section{Table 1. Four groups of different glucose levels}

\begin{tabular}{|lll|}
\hline Group & \multicolumn{2}{c|}{ Glucose Level (mmol/L) } \\
\cline { 2 - 3 } & $1^{\text {st }}$ day & $2^{\text {nd }-14^{\text {th }} \text { day }}$ \\
\hline Stable & $<7$ & Always $<10$ \\
\hline Unstable & $<7$ & More than once $\geq 10$ \\
\hline Well-controlled & $\geq 7$ & Always $<10$ \\
\hline Bad-controlled & $\geq 7$ & More than once $\geq 10$ \\
\hline
\end{tabular}

\subsection{Statistical analysis}

All statistical analyses were performed using the Statistical Package for the Social Sciences (SPSS version 21.0 for Windows, SPSS, Chicago). Categorical variables were compared using Pearson Chi-square analysis or Fisher's exact test. Continuous variables were compared using the ANOVA test or the Kruskal-Wallis rank-sum test. Multivariate logistic regression analysis was performed to determine the effects of the variables on 30 days mortality or $\mathrm{DCl}$. All statistical tests were two-tailed, and $p<0.05$ was considered to be statistically significant.

\section{Results}

3.1 Basic data, Biochemistry tests, Neuroimages, 30 days mortality and DCl occurrence (Table 2) 
For biochemistry tests, the patients whose average 14-day glucose were bad-controlled have some commons, such as higher Age (59.09 \pm 11.95$)$, Diabetes history, higher SBP (162.91 \pm 20.85$)$ when admission, relatively higher BUN (5.50 \pm 1.80$)$, higher HA1c (7.12 \pm 1.65$),$ BGV $(0.32 \pm 0.11)$, higher Hunt-Hess Score (2.59 \pm 0.97$)$, lower GCS Score (10.68 \pm 4.23$)$, Hydrocephalus in Forth ventricle, Third ventricle and Lateral ventricles. Bad controlled group has the highest mortality (15.91\%), then unstable (13.04\%), well-controlled (6.45\%), and stable group (1.42\%). The unstable group has the highest incidence of $\mathrm{DCl}(39.13 \%)$, then bad-controlled (29.55\%), the stable (17.92\%) and wellcontrolled (17.74\%).

Table 2. Basic data, Biochemistry tests, Neuroimages, 30-days mortality and DCl occurrence 


\begin{tabular}{|c|c|c|c|c|c|c|}
\hline & $\begin{array}{l}\text { stable } \\
(n=212)\end{array}$ & $\begin{array}{l}\text { unstable } \\
(n=23)\end{array}$ & $\begin{array}{l}\text { Well-controlled } \\
(n=62)\end{array}$ & $\begin{array}{l}\text { Bad-controlled } \\
(n=44)\end{array}$ & $F / x^{2}$ & $\mathbf{P}$ \\
\hline Age & $53.70 \pm 12.50$ & $56.65 \pm 11.69$ & $59.19 \pm 12.19$ & $59.09 \pm 11.95$ & 4.673 & 0.003 \\
\hline Sex & & & & & 7.812 & 0.050 \\
\hline Male & $103(48.58)$ & $9(39.13)$ & $18(29.03)$ & 18(40.91) & & \\
\hline Female & $109(51.42)$ & $14(60.87)$ & $44(70.97)$ & $26(59.09)$ & & \\
\hline Diabetes & 12(5.66) & $3(13.04)$ & $8(12.90)$ & $29(65.91)$ & 102.808 & $<0.001$ \\
\hline Hypertension & 119(56.13) & 17(73.91) & $40(64.52)$ & $32(72.73)$ & 6.589 & 0.086 \\
\hline SBP-adm. & $148.03 \pm 21.26$ & $155.83 \pm 17.07$ & $153.81 \pm 24.91$ & $162.91 \pm 20.85$ & 6.412 & $<0.001$ \\
\hline DBP-adm & $86.84 \pm 13.63$ & $87.74 \pm 12.90$ & $85.90 \pm 16.06$ & $86.89 \pm 15.27$ & 0.114 & 0.952 \\
\hline Temp.-adm & $36.76 \pm 0.79$ & $36.81 \pm 0.52$ & $36.91 \pm 0.56$ & $36.95 \pm 0.42$ & 1.384 & 0.248 \\
\hline \multicolumn{7}{|l|}{ Lab tests } \\
\hline AST & $25.39 \pm 17.11$ & $26.97 \pm 13.18$ & $26.30 \pm 15.08$ & $25.48 \pm 12.38$ & 0.107 & 0.956 \\
\hline ALT & $25.56 \pm 17.34$ & $26.47 \pm 14.42$ & $25.00 \pm 14.49$ & $23.43 \pm 10.41$ & 0.270 & 0.847 \\
\hline BUN & $4.72 \pm 1.43$ & $5.33 \pm 1.62$ & $5.11 \pm 1.54$ & $5.50 \pm 1.80$ & 4.215 & 0.006 \\
\hline $\mathrm{Cr}$ & $56.11 \pm 16.09$ & $53.62 \pm 18.79$ & $54.10 \pm 18.24$ & $60.56 \pm 19.13$ & 1.443 & 0.230 \\
\hline D-dimer & $2.99 \pm 4.00$ & $3.80 \pm 3.48$ & $4.71 \pm 7.37$ & $3.25 \pm 4.13$ & 2.075 & 0.103 \\
\hline BNP & $156.84 \pm 305.31$ & $131.62 \pm 147.94$ & $177.76 \pm 312.28$ & $123.59 \pm 154.63$ & 0.325 & 0.807 \\
\hline EF & $64.52 \pm 5.96$ & $64.26 \pm 4.39$ & $64.50 \pm 3.68$ & $65.79 \pm 4.84$ & 0.591 & 0.622 \\
\hline HA1c & $5.44 \pm 0.40$ & $5.55 \pm 0.37$ & $5.76 \pm 0.68$ & $7.12 \pm 1.65$ & 57.543 & $<0.001$ \\
\hline BGV & $0.13 \pm 0.06$ & $0.32 \pm 0.11$ & $0.15 \pm 0.06$ & $0.23 \pm 0.10$ & 56.862 & $<0.001$ \\
\hline Hunt-Hess & $2.01 \pm 0.88$ & $2.30 \pm 1.02$ & $2.52 \pm 0.97$ & $2.59 \pm 0.97$ & 8.225 & $<0.001$ \\
\hline CT-Fisher & $2.24 \pm 0.83$ & $2.39 \pm 0.72$ & $2.34 \pm 0.85$ & $2.55 \pm 0.70$ & 1.936 & 0.124 \\
\hline GCS & $13.57 \pm 2.72$ & $12.43 \pm 3.59$ & $11.34 \pm 4.04$ & $10.68 \pm 4.23$ & 14.144 & $<0.001$ \\
\hline Hydrocephalus & $68(32.08)$ & $11(47.83)$ & $26(41.94)$ & $19(43.18)$ & 4.706 & 0.195 \\
\hline Forth ventricle & $47(22.17)$ & $12(52.17)$ & $20(32.26)$ & 12(27.27) & 10.839 & 0.013 \\
\hline Third ventricle & 17(8.02) & $8(34.78)$ & $14(22.58)$ & $10(22.73)$ & 20.633 & $<0.001$ \\
\hline $\begin{array}{l}\text { Lateral } \\
\text { ventricles }\end{array}$ & 103(48.58) & 18(78.26) & $40(64.52)$ & $30(68.18)$ & 13.832 & 0.003 \\
\hline $\begin{array}{l}\text { Quadrigeminal } \\
\text { cistern }\end{array}$ & 17(8.02) & $2(8.70)$ & $7(11.29)$ & 7(15.91) & 2.832 & 0.418 \\
\hline $\begin{array}{l}\text { Cisterna } \\
\text { amibiens }\end{array}$ & $65(30.66)$ & $7(30.43)$ & 19(30.65) & $22(50.00)$ & 6.483 & 0.090 \\
\hline
\end{tabular}




\begin{tabular}{|lllllll|}
$\begin{array}{l}\text { Suprasellar } \\
\text { cistern }\end{array}$ & $115(54.25)$ & $13(56.52)$ & $43(69.35)$ & $31(70.45)$ & 7.179 & 0.066 \\
\hline Basilar cistern & $130(61.32)$ & $14(60.87)$ & $45(72.58)$ & $34(77.27)$ & 5.921 & 0.116 \\
\hline $\begin{array}{l}\text { Lateral fissure } \\
\text { cistern }\end{array}$ & $164(77.36)$ & $17(73.91)$ & $47(75.81)$ & $40(90.91)$ & 4.744 & 0.191 \\
\hline 30 d mortality & $3(1.42)$ & $3(13.04)$ & $4(6.45)$ & $7(15.91)$ & 20.224 & $<0.001$ \\
\hline DCl & $38(17.92)$ & $9(39.13)$ & $11(17.74)$ & $13(29.55)$ & 8.144 & 0.043 \\
\hline
\end{tabular}

3.2 Multivariate logistic regression analysis, 30 days Mortality and DCI

Taking the 30 days Mortality or $\mathrm{DCl}$ occurrence during hospitalization as the dependent variable respectively, and age, gender, SBP at admission, history of Diabetes Mellitus, BUN, HA1 c, BGV, Hunt-Hess GCS, Hydrocephalus in Forth ventricle, Third ventricle and Lateral ventricles as independent variable, multivariate Logistic regression analysis showed that unstable and bad-controlled group have higher 30 days Mortality and DCl incidence (Table 3.)

Table 3. Multivariate Logistic Regression Analysis for 30 days Mortality and DCI 


\begin{tabular}{|c|c|c|c|c|c|}
\hline \multicolumn{6}{|l|}{ Mortality } \\
\hline & & Crude Results & & Adjusted Resultst & \\
\hline Group & $N(\%)$ & $\begin{array}{l}\text { Hazard Ratio } \\
(95 \% \mathrm{Cl})\end{array}$ & P-value & $\begin{array}{l}\text { Hazard Ratio } \\
(95 \% \mathrm{Cl})\end{array}$ & P-value \\
\hline Stable & $3(1.4)$ & 1.000 & & 1.000 & \\
\hline Unstable & $3(13.0)$ & 7.541(1.507-37.729) & 0.014 & $14.033(1.971-99.921)$ & 0.008 \\
\hline Well-controlled & $4(6.5)$ & $4.939(1.105-22.076)$ & 0.037 & $4.881(0.780-30.534)$ & 0.090 \\
\hline Bad-controlled & $7(15.9)$ & $10.487(2.694-40.818)$ & 0.001 & 19.723(3.597-108.143) & 0.001 \\
\hline \multicolumn{6}{|l|}{$\mathrm{DCl}$} \\
\hline & & Crude Results & & Adjusted Resultst & \\
\hline Group & $N(\%)$ & $\begin{array}{l}\text { Odds Ratio } \\
\text { (95\% Cl) }\end{array}$ & P-value & $\begin{array}{l}\text { Odds Ratio } \\
\text { (95\% Cl) }\end{array}$ & P-value \\
\hline Stable & $38(17.9)$ & 1.000 & & 1.000 & \\
\hline Unstable & $9(39.1)$ & $2.944(1.187-7.298)$ & 0.020 & $6.032(1.941-18.747)$ & 0.002 \\
\hline Well-controlled & $11(17.7)$ & $0.988(0.471-2.070)$ & 0.974 & $1.115(0.497-2.502)$ & 0.792 \\
\hline Bad-controlled & $13(29.53)$ & $1.920(0.919-4.011)$ & 0.083 & $2.889(1.247-6.691)$ & 0.013 \\
\hline
\end{tabular}

† Adjusted for age, gender, SBP at admission, history of Diabetes Mellitus, BUN, HA1c, BGV, Hunt-Hess GCS, Hydrocephalus in Forth ventricle, Third ventricle and Lateral ventricles

rom aneurysm SAH were included. People with SAH caused by vasculitis, trauma, or rupture of an arteriovenous malformation was excluded. There were 341 subjects (148 males, $43.4 \%$; 193 females, $56.6 \%$ ) were enrolled in this study. We have two experts to evaluate the occurrence of $\mathrm{DCl}$, the Kappa value was 0.91 between the two experts. This retrospective study was approved by the Ethics Committee at the Tiantan Hospital in Beijing, in accordance with the Declaration of Helsinki. Our study was approved to collect data without requiring patient informed consent under the Waived Consent from the Ethics Committee at the Tiantan Hospital in Beijing (Ethics Approval Number: KY2019-060).

\subsection{Clinical information}

The following baseline characteristics were analyzed based on the data collected during the period of admission: sex, age, diabetes mellitus (DM), hypertension, glucose level, initial Glasgow Coma Scale (GCS) score, systolic blood pressure (SBP), diastolic blood pressure (DBP), temperature, treatment, surgery, mortality information and dates of death. Several indexes were collected as follows: AST, ALT, BUN, creatinine (Cr), D-dimer, BNP, EF, hemoglobin A1c (HA1c), blood glycemic variability (BGV), Hunt Hess, CT Fisher, hydrocephalus (Fourth ventricle, Third ventricle, Lateral ventricle, Quadrigeminal cistern, Ambiens cisterna, Suprasellar cistern, Sylvian fissure, Basilar cistern). The first day blood glucose level was taken immediately before any glucose management when admission. Then the other 13 days blood sample was taken around 6:00 am every morning as fasting blood glucose. These 14 days glucose value after SAH were collected and was divided into four groups - stable, unstable, 
well-controlled and bad controlled (Table 1). BGV was measured as glycated albumin/glycosylated hemoglobin ratio[13].

Table 1

Four groups of different glucose levels

\begin{tabular}{|lll|}
\hline Group & \multicolumn{2}{l|}{ Glucose Level $(\mathrm{mmol} / \mathrm{L})$} \\
\cline { 2 - 3 } & 1st day & 2nd $-\mathbf{1 4 t h}$ day \\
\hline Stable & $<7$ & Always $<10$ \\
\hline Unstable & $<7$ & More than once $\geq 10$ \\
\hline Well-controlled & $\geq 7$ & Always $<10$ \\
\hline Bad-controlled & $\geq 7$ & More than once $\geq 10$ \\
\hline
\end{tabular}

\subsection{Statistical analysis}

All statistical analyses were performed using the Statistical Package for the Social Sciences (SPSS version 21.0 for Windows, SPSS, Chicago). Categorical variables were compared using Pearson Chi-square analysis or Fisher's exact test. Continuous variables were compared using the ANOVA test or the Kruskal-Wallis rank-sum test. Multivariate logistic regression analysis was performed to determine the effects of the variables on 30 days mortality or $\mathrm{DCl}$. All statistical tests were two-tailed, and $p<0.05$ was considered to be statistically significant.

\section{Discussion}

In this retrospective study, several risk factors were found to result in bad-controlled or unstable glucose during admission in NCU, such as older age, history of Diabetes Mellitus, higher SBP, BUN, HA1c, BGV and Hunt-Hess Score, lower GCS score, and Hydrocephalus in Forth ventricle, Third ventricle and Lateral ventricles. Subsequently, multivariate logistic regression analysis also showed that these factors have direct association with higher 30 days mortality and $\mathrm{DCl}$ incidence in patients with bad-controlled and unstable glucose level during NCU.

Hyperglycemia after SAH onset was induced by multiple responsive system, such as the activation of hypothalamic-pituitary-adrenal axis (HPA), the activation of sympathetic autonomic nervous system[14], the release of HPA related cytokines after increased inflammatory response[15], and the reduction of hepatic gluconeogenesis and increase of insulin sensitivity regulated by hypothalamus[16]. Clinically, mean glucose of patients admitted within 72 hours after aSAH is around $9 \mathrm{mmol} / \mathrm{L}$, and still exceeding 7-8 mmol/L 1-2 weeks later[2]. Several researches revealed that hyperglycemia on admission or persistent hyperglycemia, occurred in 30$100 \%$ SAH patients, was involved in DCI and short- or long-term poor outcomes[17]. Similarly, our study found that average glucose and BGV was higher in patients who suffered DCl or had high mortality. In addition, HA1c, used as an evaluation of average blood sugar levels over a period of 2-3 months, was proven being associated with DCl and poor outcome in the present study. Different from what we found, collecting HA1c of 87 aSAH patients within $72 \mathrm{~h}$ of admission, Beseoglu et al. showed that $\mathrm{HA} 1 \mathrm{c}$ had little significant correlation with $\mathrm{DCl}$ occurrence or DCl related infarction by using correlation analysis[18]. However, the number of cases recruited was low in the study which could negatively influence statistical power. Therefore, although HA1c was proven having no association with rupture of aneurysms[19], whether HA1c could be a predictor of DCl and unfavorable outcome in aSAH still requires more evidence. 
Intensive insulin therapy (IIT) was used worldwide in both surgical and general ICU, even though it was associated with severe hypoglycemia and subsequent poor clinical outcome. However, the value of IIT used in NCU patients was still controversial because those people, due to brain injury, were always highly susceptible to either hyperglycemia or hypoglycemia. Especially, acute injured brain required constant glucose supply in order to satisfy brain metabolism while hypoglycemia induced by IIT might lead to energy failure[20]. Different from in general ward, patients in NCU with critical brain injuries whose glucose level should have been monitored regularly and frequently, hence, rigorous and fine-tuning glucose management can be realized and be benefit to clinical outcome. Up to now, most research mainly discussed about optimal glycemia control in SAH patients in order to obtain favorable outcome. However, both the optimal pending value of hyperglycemia or the optimal cutoff value of hypoglycemia was still inconclusive. Two glucose control regimens from two single center before-after studies, 5.0 - $6.7 \mathrm{mmol} / \mathrm{L}$ and $4.4-7.8 \mathrm{mmol} / \mathrm{L}$, failed to improve clinical outcome or reduce mortality of SAH, but was associated with incidence of hypoglycemia [21, 22]. Similarly, another control regimen $(4.4-6.7)$ from a single center RCT study was proven to reduce infection rate but still no influence on postoperative vasospasm, neurological outcomes and mortality rates [23]. These evidences showed that narrowing glucose fluctuation doesn't seem favorable to SAH outcomes. In this retrospective study, we divided glucose level into four groups and find that $\mathrm{DCl}$ incidence was higher in those patients whose glucose level was greater than $10 \mathrm{mmol} / \mathrm{L}$ at least once during 14 consecutive days, no matter whether glucose at the first day was more than $7 \mathrm{mmol} / \mathrm{L}$ or not. Thus, 10 $\mathrm{mmol} / \mathrm{L}$ seemed as an important upper limit that was associated with incidence of $\mathrm{DCl}$ in SAH patients, while 7 $\mathrm{mmol} / \mathrm{L}$ was not for sure to be optimal lower limit because we tended to stop insulin treatment to avoid hypoglycemia when every two-hour fasting glucose level was tested lower than $5 \mathrm{mmol} / \mathrm{L}$ in NCU.

BGV refers to swing in blood glucose level and was highly suggested to be an important key determinant of vascular damage [24]. Several studies showed BGV was possibly associated with cerebral infarction, mortality and even clinical poor outcomes in central nervous system diseases. Using standard deviation (SD) as daily glycemic variability (GV), a retrospective study observed 28 patients with SAH and revealed that systematic GV was associated with cerebral metabolic distress and hospital mortality in SAH[5]. Although no reference to GV, another study showed that acute reduction of glucose level, in spite of being within normal range, was related to brain energy metabolic crisis in poor grade SAH patients [6]. Similarly, our present study found that BGV was associated with occurrence of $\mathrm{DCl}$ and high mortality within 30 days admission.

Up to now, there was still no consensus on the relationship of age and DCl incidence. Some studies showed that relatively young people have greater risk of occurring $\mathrm{DCl}$. Using univariate analysis on 463 aSAH patients with an overall DCl incidence of $21 \%$, Lee et al found that age between $40-59$, higher modified Fisher grade and aneurysm rupture in anterior circulation could be more accurate predictors of DCl risk [25]. Another earlier study that included 3567 aSAH patients, using multivariate analysis, revealed that symptomatic vasospasm has significant association with age 40 to 59 years[26]. Most theory thought that, compared to younger ones, senescent intracranial arteries were so stiffer and less elastic, due to thicker vessel walls, higher thickness-to-radius ratio and more collagen fiber, that they are more resistant to vasospasm [25]. However, the mechanism is still unclear. Similarly, this present study showed older patients tend to have $\mathrm{DCl}$ and higher 30 days mortality. Contrarily, in a systematic review, 6 studies included showed that there was no difference with risk of DCl between young and old patients. Thus, different theories came from these results thought young people might have stronger resilient to ischemia and better autoregulation [25].

\section{Conclusions}

Page $10 / 14$ 
We showed that unstable or bad-controlled glucose fluctuation was associated with DCI incidence and high mortality in SAH patients who are critically ill and admitted in NCU. Several risk factors, such as age, history of Diabetes Mellitus, SBP, BUN, HA1c, BGV, Hunt-Hess Score, GCS score, and Hydrocephalus in Forth ventricle, Third ventricle and Lateral ventricle, probably led to unstable and bad-controlled glucose lever during admission, and eventually $\mathrm{DCl}$ and death.

\section{List Of Abbreviations}

\begin{tabular}{ll} 
DCI & delayed cerebral infraction \\
\hline aSAH & aneurysmal subarachnoid hemorrhage \\
\hline NCU & neurocritical unit \\
\hline TBI & traumatic brain injury \\
\hline DM & diabetes mellitus \\
\hline GCS & glasgow coma scale \\
\hline SBP & systolic blood pressure \\
\hline DBP & diastolic blood pressure \\
\hline Cr & creatinine \\
\hline HA1c & hemoglobin A1c \\
\hline BGV & blood glycemic variability \\
\hline AST & glutamic oxaloacetic transaminase \\
\hline ALT & glutamic pyruvic transaminase \\
\hline BUN & urea nitrogen \\
\hline EF & ejection fraction \\
\hline HPA & hypothalamic-pituitary-adrenal axis \\
\hline IIT & intensive insulin therapy \\
\hline GV & glycemic variability \\
\hline D
\end{tabular}

\section{Declarations}

Ethics approval and consent to participate: This retrospective study was approved by the Ethics Committee at the Tiantan Hospital in Beijing. Our study was approved to collect data without requiring patient informed consent under the Waived Consent from the Ethics Committee at the Tiantan Hospital in Beijing (Ethics Approval Number: KY2019-060). Patients privacy and data confidentiality were ensured.

Consent for publication:Not applicable.

Availability of data and material:The datasets during and/or analyzed during the current study are available from the corresponding author on reasonable request. 
Conflict of Interest: The authors declare that they have no conflict of interest.

Funding: None.

Authors' contributions:

QW and LT:conceived and designed the study and drafted and revise the manuscript for important intellectual content.

LZ and LL:conceived and designed the study.

DM: given final approval of the version and financial support.

Acknowledgements:The authors thank all participating colleagues, nurses, and imaging and laboratory technicians.

\section{References}

1. Okazaki T, Kuroda Y: Aneurysmal subarachnoid hemorrhage: intensive care for improving neurological outcome. Journal of intensive care 2018, 6:28.

2. Kruyt ND, Biessels GJ, DeVries JH, Luitse MJ, Vermeulen M, Rinkel GJ, Vandertop WP, Roos YB: Hyperglycemia in aneurysmal subarachnoid hemorrhage: a potentially modifiable risk factor for poor outcome. Journal of cerebral blood flow and metabolism : official journal of the International Society of Cerebral Blood Flow and Metabolism 2010, 30(9):1577-1587.

3. Abulhasan YB, Alabdulraheem N, Schiller I, Rachel SP, Dendukuri N, Angle MR, Frenette C: Health CareAssociated Infections after Subarachnoid Hemorrhage. World neurosurgery 2018, 115:e393-e403.

4. Steiner T, Juvela S, Unterberg A, Jung C, Forsting M, Rinkel G, European Stroke O: European Stroke Organization guidelines for the management of intracranial aneurysms and subarachnoid haemorrhage. Cerebrovascular diseases 2013, 35(2):93-112.

5. Kurtz P, Claassen J, Helbok R, Schmidt J, Fernandez L, Presciutti M, Stuart RM, Connolly ES, Lee K, Badjatia N et al: Systemic glucose variability predicts cerebral metabolic distress and mortality after subarachnoid hemorrhage: a retrospective observational study. Critical care 2014, 18(3):R89.

6. Helbok R, Schmidt JM, Kurtz P, Hanafy KA, Fernandez L, Stuart RM, Presciutti M, Ostapkovich ND, Connolly ES, Lee $\mathrm{K}$ et al: Systemic glucose and brain energy metabolism after subarachnoid hemorrhage. Neurocritical care 2010, 12(3):317-323.

7. Foreman B: The Pathophysiology of Delayed Cerebral Ischemia. Journal of clinical neurophysiology : official publication of the American Electroencephalographic Society 2016, 33(3):174-182.

8. Jafri H, Diringer MN, Allen M, Zazulia AR, Zipfel GJ, Dhar R: Burden of cerebral hypoperfusion in patients with delayed cerebral ischemia after subarachnoid hemorrhage. Journal of neurosurgery 2019:1-8.

9. de Rooij NK, Rinkel GJ, Dankbaar JW, Frijns CJ: Delayed cerebral ischemia after subarachnoid hemorrhage: a systematic review of clinical, laboratory, and radiological predictors. Stroke 2013, 44(1):43-54.

10. van Donkelaar CE, Dijkland SA, van den Bergh WM, Bakker J, Dippel DW, Nijsten MW, van der Jagt M: Early Circulating Lactate and Glucose Levels After Aneurysmal Subarachnoid Hemorrhage Correlate With Poor 
Outcome and Delayed Cerebral Ischemia: A Two-Center Cohort Study. Critical care medicine 2016, 44(5):966972.

11. Parsons MW, Barber PA, Desmond PM, Baird TA, Darby DG, Byrnes G, Tress BM, Davis SM: Acute hyperglycemia adversely affects stroke outcome: a magnetic resonance imaging and spectroscopy study. Annals of neurology 2002, 52(1):20-28.

12. McGirt MJ, Woodworth GF, Ali M, Than KD, Tamargo RJ, Clatterbuck RE: Persistent perioperative hyperglycemia as an independent predictor of poor outcome after aneurysmal subarachnoid hemorrhage. Journal of neurosurgery 2007, 107(6):1080-1085.

13. Suh S, Kim JH: Glycemic Variability: How Do We Measure It and Why Is It Important? Diabetes \& metabolism journal 2015, 39(4):273-282.

14. Gauna $\mathrm{C}$, van den Berghe $\mathrm{GH}$, van der Lely AJ: Pituitary function during severe and life-threatening illnesses. Pituitary 2005, 8(3-4):213-217.

15. Hendryk S, Jarzab B, Josko J: Increase of the IL-1 beta and IL-6 levels in CSF in patients with vasospasm following aneurysmal SAH. Neuro endocrinology letters 2004, 25(1-2):141-147.

16. Schwartz MW, Porte D, Jr.: Diabetes, obesity, and the brain. Science 2005, 307(5708):375-379.

17. Wartenberg KE: Update on the Management of Subarachnoid Hemorrhage. Future Neurology 2013, 8(2):205224.

18. Beseoglu K, Steiger HJ: Elevated glycated hemoglobin level and hyperglycemia after aneurysmal subarachnoid hemorrhage. Clinical neurology and neurosurgery 2017, 163:128-132.

19. Can A, Castro VM, Yu S, Dligach D, Finan S, Gainer VS, Shadick NA, Savova G, Murphy S, Cai T et al: Antihyperglycemic Agents Are Inversely Associated With Intracranial Aneurysm Rupture. Stroke 2018, 49(1):3439.

20. Godoy DA, Di Napoli M, Rabinstein AA: Treating hyperglycemia in neurocritical patients: benefits and perils. Neurocritical care 2010, 13(3):425-438.

21. Thiele RH, Pouratian N, Zuo Z, Scalzo DC, Dobbs HA, Dumont AS, Kassell NF, Nemergut EC: Strict glucose control does not affect mortality after aneurysmal subarachnoid hemorrhage. Anesthesiology 2009, 110(3):603-610.

22. Latorre JG, Chou SH, Nogueira RG, Singhal AB, Carter BS, Ogilvy CS, Rordorf GA: Effective glycemic control with aggressive hyperglycemia management is associated with improved outcome in aneurysmal subarachnoid hemorrhage. Stroke 2009, 40(5):1644-1652.

23. Bilotta F, Spinelli A, Giovannini F, Doronzio A, Delfini R, Rosa G: The effect of intensive insulin therapy on infection rate, vasospasm, neurologic outcome, and mortality in neurointensive care unit after intracranial aneurysm clipping in patients with acute subarachnoid hemorrhage: a randomized prospective pilot trial. Journal of neurosurgical anesthesiology 2007, 19(3):156-160.

24. Standl E, Schnell O, Ceriello A: Postprandial hyperglycemia and glycemic variability: should we care? Diabetes care 2011, 34 Suppl 2:S120-127.

25. Lee H, Perry JJ, English SW, Alkherayf F, Joseph J, Nobile S, Zhou LL, Lesiuk H, Moulton R, Agbi C et al: Clinical prediction of delayed cerebral ischemia in aneurysmal subarachnoid hemorrhage. Journal of neurosurgery 2018:1-8.

26. Macdonald RL, Rosengart A, Huo D, Karrison T: Factors associated with the development of vasospasm after planned surgical treatment of aneurysmal subarachnoid hemorrhage. Journal of neurosurgery 2003, 
99(4):644-652.

\section{Supplementary Files}

This is a list of supplementary files associated with this preprint. Click to download.

- Highlights.doc 